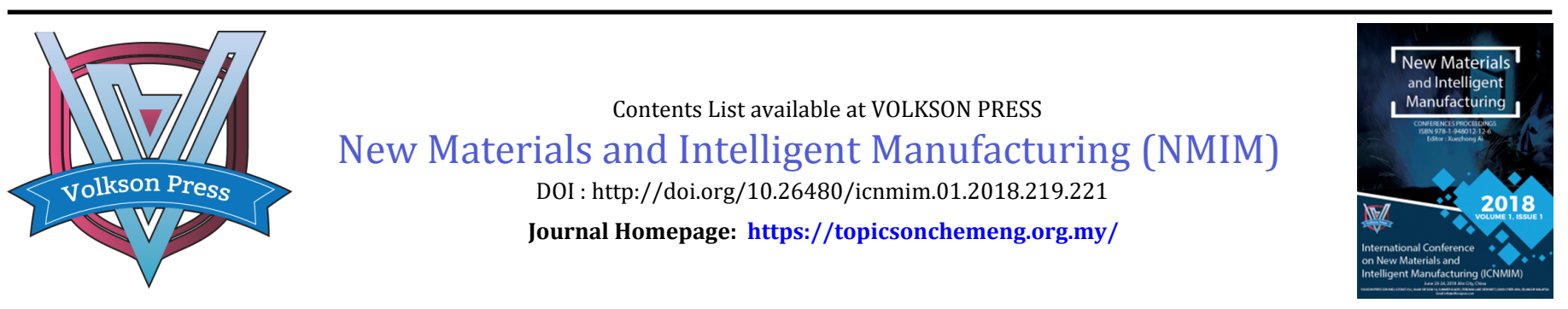

ISBN: 978-1-948012-12-6

\title{
PRELIMINARY STUDY ON IMMOBILIZATION TECHNOLOGY OF TIO2 PHOTO CATALYST
}

\author{
Yang Zhao-xu, Zhang Jing-jing, Duan Cheng-long, Chen Chen, Gong Yan, Li Ying-hua* \\ College of Resources and Environmental Engineering, Jilin Institute of Chemical Technology, Jilin Jilin 132022. \\ *Corresponding Author Email: 414336044@qq.com
}

This is an open access article distributed under the Creative Commons Attribution License, which permits unrestricted use, distribution, and reproduction in any medium, provided the original work is properly cited

\section{ARTICLE DETAILS}

\section{Article History:}

Received 26 June 2018 Accepted 2 July 2018

Available online 1 August 2018

\section{ABSTRACT}

$\mathrm{TiO}_{2}$ was used as photo catalyst to treat organic effluent firstly. Methyl orange solution was utilized as simulated wastewater for optimizing conditions of the reaction. That is: reaction time as $60 \mathrm{~min}$, solution concentration as $50 \mathrm{mg} / \mathrm{L}$, and amount of catalyst as $0.02 \mathrm{~g}$. $\mathrm{TiO}_{2}$ was then immobilized on diatomite (so-called $\mathrm{TiO}_{2} /$ diatomite composite materials) for treatment of organic effluent. The reaction mechanism was studied. The results showed that $\mathrm{TiO}_{2}$ played as a good photocatalyst, diatomite possessed a certain adsorption capacity for methyl orange removal. The removal rate of $100 \%$ for advanced treatment of actual organic wastewater was obtained by using this composite material.

\section{KEYWORDS}

$\mathrm{TiO}_{2}$, immobilization, diatomite, composite material, photocatalysis.

\section{INTRODUCTION}

$\mathrm{TiO}_{2}$ is recognized as the most effective semiconductor catalyst. Due to the low cost, large supply channels, high performance, and safety that are recognized through long-term social verification. There is significant potential in industrial-scale engineering applications for water/atmosphere purification [1-4]. However, in practical applications, there're many problems on suspended $\mathrm{TiO}_{2}$ photocatalyst application, such as easy aggregation, difficulty in recovery, and secondary pollution caused by self-dispersion. Therefore, loading and fixing it on a suitable carrier is the key to the industrial processing application of the heterogeneous photocatalytic technology.

The diatomite has a diatom shell structure, large specific surface area, strong adsorption, high porosity, high temperature resistance and other excellent characteristics [5]. Its large specific surface area allows $\mathrm{TiO}_{2}$ particles uniformly loaded on. On the other hand, its good adsorption properties make organic substances adsorbed on the surface, which increases the contact between organic matter and $\mathrm{TiO}_{2}$ and probability increases the photocatalytic efficiency [6-10].

The preparation methods of $\mathrm{TiO}_{2}$ /diatomite catalyst mainly include impregnation method, precipitation method and chemical deposition method $[11,12]$. In this paper, $\mathrm{TiO}_{2}$ /diatomite composites were prepared by coating method, and characterized by SEM and XRD. The catalytic degradation of organic wastewater was investigated. The influencing factors of the preparation method were discussed.

\section{EXPERIMENT}

\subsection{Instruments and Reagents}

$\mathrm{TiO}_{2}$ (Degussa P25, Germany); Diatomaceous earth (filter with $0.3 \mathrm{~mm}$ sieve for backup); Non-woven fabrics (purchased by Jilin Xiongfa Technology Co., Ltd., washed and dried repeatedly before use); Methyl orange (A.P.); The water sample of experimental wastewater comes from the second settling tank of a sewage treatment plant in Jilin city.BL-GHX-V photochemical reaction instrument (Shanghai Billon); UVmini-1240 spectrophotometer (Shimadzu Corporation, Japan); Sievers Innov0x laboratory TOC analyzer (US PE company).

\subsection{Preparation of $\mathrm{TiO}_{2} /$ Diatomite Composites}

First, a $10 \mathrm{wt} \% \mathrm{TiO}_{2} /$ diatomite homogenate was prepared and ultrasonically oscillated for 30 minutes. Then the homogenate was coated on treated non-woven fabric $\left(15 \mathrm{~cm}^{*} 10 \mathrm{~cm}\right)$ for several times. After each coating, it was dried at $105^{\circ} \mathrm{C}$ for 1 hour to obtain a $\mathrm{TiO}_{2} /$ diatomite composite material.

\section{RESULTS AND DISCUSSION}

\subsection{Optimization of Photocatalytic Reaction Conditions}

In order to examine the combined effects of all main factors, an orthogonal optimization experiment of L9(3) was conducted. Here factor A is reaction time of $20 \mathrm{~min}, 40 \mathrm{~min}$ and $60 \mathrm{~min}$; factor $\mathrm{B}$ is the concentration of methyl orange with $40 \mathrm{mg} / \mathrm{L}, 50 \mathrm{mg} / \mathrm{L}$ and $60 \mathrm{mg} / \mathrm{L}$; and factor $C$ is the amount of P25, which were $0.01 \mathrm{~g}, 0.02 \mathrm{~g}$ and $0.03 \mathrm{~g}$. The experimental results are shown in Table 1: 
Table 1: Orthogonal test data

\begin{tabular}{llllll}
\hline No. & Factor A & Blank & Factor B & Factor C & $\begin{array}{c}\text { Degradation } \\
(\%)\end{array}$ \\
\hline 1 & 1 & 1 & 1 & 1 & 85.0 \\
2 & 1 & 2 & 2 & 2 & 86.5 \\
3 & 1 & 3 & 3 & 3 & 86.7 \\
4 & 2 & 1 & 2 & 3 & 92.5 \\
5 & 2 & 2 & 3 & 1 & 86.7 \\
6 & 2 & 3 & 1 & 2 & 90.0 \\
7 & 3 & 1 & 3 & 2 & 97.8 \\
8 & 3 & 2 & 1 & 3 & 100.0 \\
9 & 3 & 3 & 2 & 1 & 95.3 \\
$\mathrm{~K}_{1}$ & 258.2 & 275.3 & 275.0 & 266.9 & \\
$\mathrm{~K}_{2}$ & 269.2 & 273.2 & 274.3 & 274.3 & 279.2 \\
$\mathrm{~K}_{3}$ & 293.1 & 271.9 & 271.2 & 12.3 & \\
$\mathrm{R}$ & 34.9 & 3.4 & 3.8 & & \\
\hline
\end{tabular}

From Table 1, it can be seen that the highest degradation rate is obtained in the condition of $\mathrm{A}_{3} \mathrm{~B}_{1} \mathrm{C}_{3}$, which is $100 \%$. According to the comparison of $R$ as $R_{A}>R_{C}>R_{B}$, the main influencing factor is the reaction time, and then are the amount of catalyst and the concentration of methyl orange solution. Further analysis of $K$ values (A column: $K_{3}>K_{2}>K_{1}$, B column: $K_{1}>$ $K_{2}>K_{3}$, C column: $K_{3}>K_{2}>K_{1}$ ), the same optimized condition of $A_{3} B_{1} C_{3}$ was derived. But the final experimental condition of $\mathrm{A}_{3} \mathrm{~B}_{2} \mathrm{C}_{2}$ was decided in consideration of financial cost, which is reaction time was $60 \mathrm{~min}$, the concentration of methyl orange was $50 \mathrm{mg} / \mathrm{L}$, and the amount of P25 was $0.02 \mathrm{~g}$.

\subsection{Effect of the adsorption of methyl orange on catalyst carrier}

\subsubsection{Adsorption kinetics}

In order to investigate the adsorption characteristics of the methyl orange solution, the absorbance of the methyl orange at different shaking times of the catalyst carrier were measured, and then converted into the removal rate.

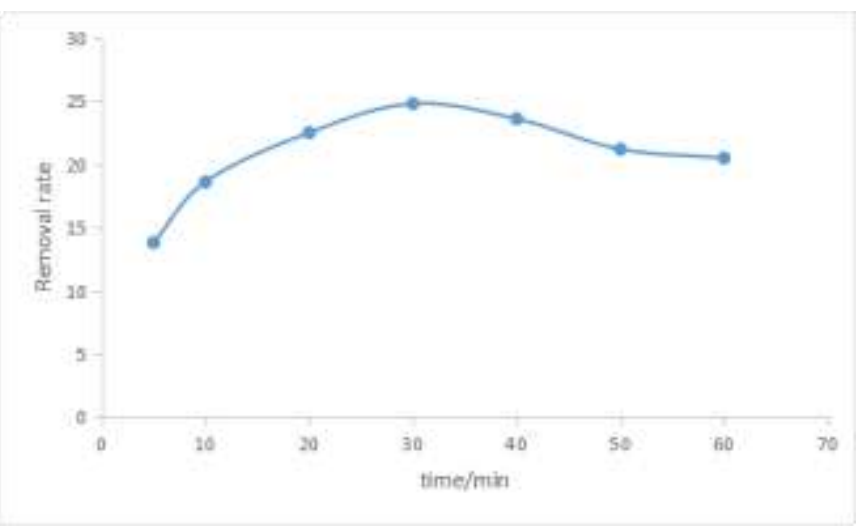

Figure 1: Removal rate of methyl orange with time

According to Figure 1, it can be seen that with the increasing of time, the removal rate gradually increases, and then reaches the maximum value of $24.8 \%$ at $30 \mathrm{~min}$. After $30 \mathrm{~min}$, the removal rate decreased a little, and then became stable. It indicates that catalyst carrier reach adsorption saturation after $30 \mathrm{~min}$.

\subsubsection{Adsorption isotherms}

According to the relationship between the adsorption amount $(\mathrm{G})$ and the equilibrium concentration (c) (Figure 2), the adsorption isotherm type for the adsorption of solution on the catalyst carrier belongs to be Freundlich type. It shows that the catalyst carrier has a certain adsorption capacity of methyl orange.

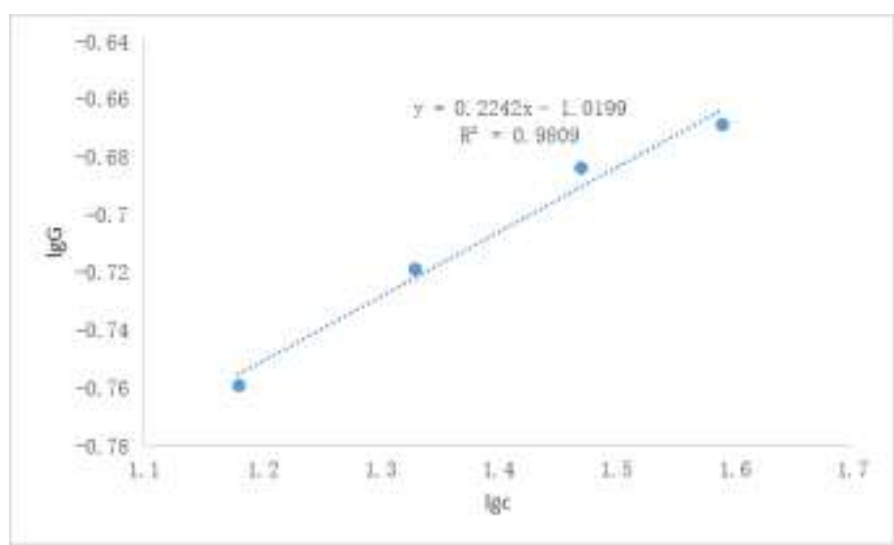

Figure 2: Adsorption isotherm of methyl orange solution by catalyst carrier

\subsection{Repeatability Test of $\mathrm{TiO}_{2} /$ Diatomite Composites}

In order to investigate the durability of the composites, a $10 \mathrm{wt} \%$ $\mathrm{TiO}_{2}$ /diatomite composite material with good removal effect was subjected to repeated experiments under the above conditions.

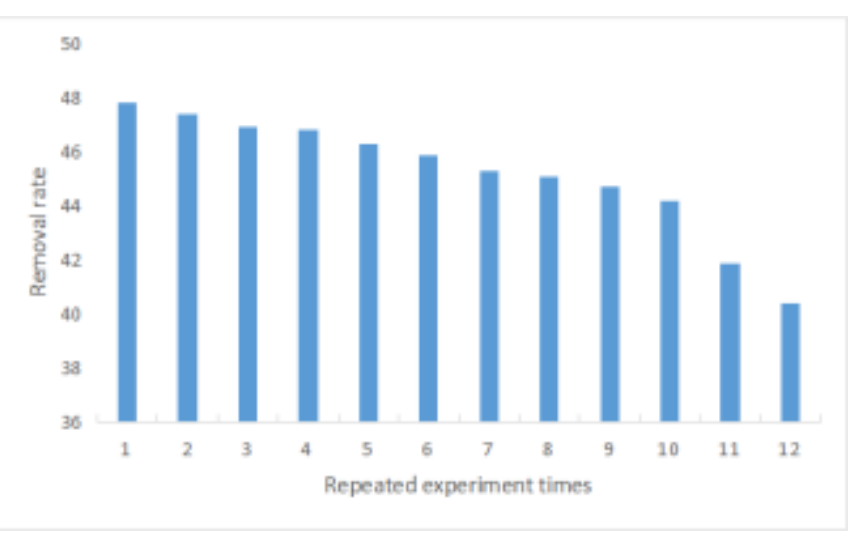

Figure 3: Repeated experiments

The results showed that the removal rate of methyl orange was slowly reduced to a certain extent beyond $44 \%$ at the former 10 times, and then reduce rapidly after 11 times. By weighing after every repeated experiment, the coated particles on the composite material were observed fallen off to a certain extent to $95.1 \%$, which may caused the reduction of organic removal efficiency.

\subsection{Characterization of $\mathrm{TiO}_{2} /$ Diatomite Composites}

Cite The Article: Yang Zhao-Xu, Zhang Jing-Jing, Duan Cheng-Long, Chen Chen, Gong Yan, Li Ying-Hua (2018). Preliminary Study On Immobilization Technology Of Tio2 Photo Catalyst . Topics in Chemical \& Material Engineering , 1(1) : 219-221. 
The SEM and XRD of the composites are shown in Figure 4 and 5, respectively.

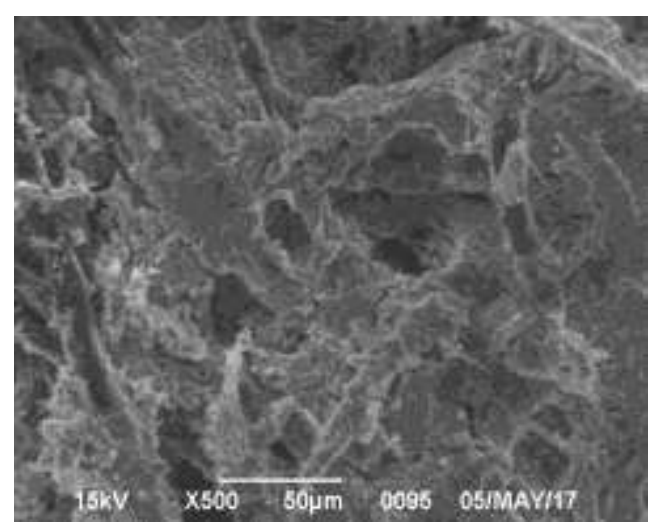

Figure 4: $\mathrm{SEM}$ of $\mathrm{TiO}_{2}$ / diatomite composite material

According to figure $4, \mathrm{TiO}_{2}$ and diatomaceous earth were evenly covered on the surface of the nonwoven fabric can be observed.

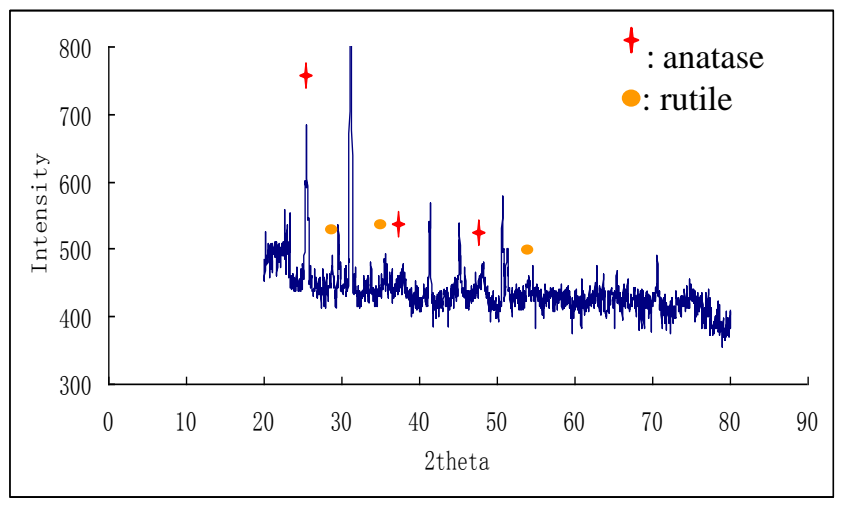

Figure 5: $\mathrm{XRD}$ of $\mathrm{TiO}_{2} /$ diatomite composite material

The characteristic peaks of anatase appeared at $2 \theta=25.325,37.841$, 48.074 , and the characteristic peaks of rutile appeared at $2 \theta=27.459$, $36.104,54.364$. The XRD pattern of the tested composite material (Figure 5) can be determined the presence of both anatase and rutile phases. It indicated that the P25 component in the composite is uniformly distributed on the surface of the support, playing the role of catalytically active center. The experimental results showed that the composite material not only has an adsorption effect but also exhibits a good photocatalytic performance, which supports the above conclusions.

\subsection{Advanced treatment of organic wastewater on $\mathrm{TiO}_{2} /$ Diatomite composite}

The $10 \% \mathrm{TiO}_{2}$ /diatomite composite material was applied for advanced treatment of the actual organic wastewater instead of methyl orange solution under the same experimental conditions. The result showed that the removal rate reached $100 \%$, which is much better than the treatment of methyl orange simulated wastewater under the same conditions (the removal rate is $46.1 \%$ ). It may be speculated that the actual organic wastewater composition is complex. Although the concentration of organic matter is similar to that of methyl orange, the adsorption and catalytic degradation of composite materials maybe changed. Further study of the composite materials should be performed to investigate the reaction mechanism.

\section{CONCLUSIONS}

In summary, the $\mathrm{TiO}_{2}$ /diatomite composite material owns both adsorption and photocatalysis effects. Organic pollutants can be adsorbed on the surface of the composite material and photocatalytic degradated to ideal content. This immobilization technology solves the recovery problem of $\mathrm{TiO}_{2}$ suspended phase and has a good application prospect. However, it is necessary for further study on the loading method and durability.

\section{REFERENCES}

[1] Fujishima, A., Rao, T.N., Tryk, D.A. 2000. Titanium dioxide photocatalysis [J]. Journal Photoch Photobio C: Photochem Review, 1 (1), $1-7$.

[2] Xin, X., Ya-fei, T. 2004. Study on Photocatalytic Degradation of Dichlorvos in Water with Activated Carbon Supported $\mathrm{TiO}_{2}$ [J]. Industrial Water \& Wastewater, 35 (1), 30-32.

[3] Cui-hua, Z., Jian-hua, C., Zhi-qiang, S. 2004. Experimental study on the influence of different carriers on the photocatalytic activity of $\mathrm{TiO}_{2}$ thin films [J]. Industrial Water \& Wastewater, 35 (4), 15-17.

[4] Wei, Z., Hui-sheng, Z. 2004. Modification of $\mathrm{TiO}_{2}$ photocatalytic film and its catalytic performance [J]. Industrial Water \& Wastewater, 35 (5), 3537.

[5] Jian-kang, D., Lin-sheng, Z., Ming-fang, X. 2007. Study on Degradation of Dimethyl Formamide by Ti02-Diatomite Composite Photocatalyst [J]. Industrial Water \& Wastewater, 38 (6), 38-40.

[6] Ying, M., Jian-bin, Q. 2001. Photocatalytic activity of $\mathrm{TiO}_{2}$ films grown on different substrates [J]. Chemosphere, 44 (5), 1087-1092.

[7] Dong-na, L., Xiao-jun, M., Xin-yan, L. 2014. Preparation and characterization of nano- $\mathrm{TiO}_{2}$ loaded bamboo-based activated carbon fibers by $\mathrm{H}_{2} \mathrm{O}$ activation [J]. Bioresources, 9 (1), 602-612.

[8] Bin, W., Guang-xin, Z., Shui-lin, Z. 2014. The effect of calcination temperature on the crystal structure and photocatalytic performance of $\mathrm{TiO}_{2}$ /diatomite [J]. Journal of Inorganic Materials, 9 (4), 382-386.

[9] Zhi-ming, S., Chun-hua, B., Shui-lin, Z. 2013. Acomparative study of different porous amorphous silica minerals supported $\mathrm{TiO}_{2}$ catalysts [J]. Applied Catalysis A: General, 458, 103-110.

[10] Kamikoa, M., Aotania, K., Suenaga, R. 2012. The Influence of Ta underlayers on the structure of $\mathrm{TiO}_{2}$ thin films deposited on an unheated glass substrate [J]. Applied Surface Science, 258 (22), 8764-8768.

[11] Furman, P., Gluszek, J., Masalski, J. 1997. Titanium dioxide film obtained using the MOCVD method on 316L steel [J]. Journal of Materials Science Letters, 16 (6), 471-472.

[12] Schwarz, J.A. 1995. Cristian contescu and adriana contescu [J]. Chemical Review, 95 (3), 477-510. 